\title{
Astragali Radix Contributes to the Inhibition of Liver Fibrosis via High-Mobility Group Box 1-Mediated Inflammatory Signaling Pathway
}

\author{
Jianxia Wen $\mathbb{D}^{1,2}$ Dan Wang $\mathbb{D}^{1,2,3}$ Jian Wang $\mathbb{D}^{2}{ }^{2}$ Ruilin Wang, ${ }^{4}$ Shizhang Wei, ${ }^{1,2}$ \\ and Yanling Zhao $\mathbb{D}^{1}$ \\ ${ }^{1}$ Department of Pharmacy, The Fifth Medical Center of Chinese PLA General Hospital, Beijing 100039, China \\ ${ }^{2}$ College of Pharmacy, Chengdu University of Traditional Chinese Medicine, Chengdu 611137, China \\ ${ }^{3}$ Chongqing Academy of Chinese Materia Medica, Chongqing 400065, China \\ ${ }^{4}$ Clinical Laboratory Medicine Center, The Fifth Medical Center of Chinese PLA General Hospital, Beijing 100039, China
}

Correspondence should be addressed to Yanling Zhao; zhaoyl2855@126.com

Received 9 January 2021; Revised 26 February 2021; Accepted 2 March 2021; Published 15 March 2021

Academic Editor: Chih-Yuan Ko

Copyright (C) 2021 Jianxia Wen et al. This is an open access article distributed under the Creative Commons Attribution License, which permits unrestricted use, distribution, and reproduction in any medium, provided the original work is properly cited.

Astragali Radix (AR), the dried root of Astragali Radix membranaceus (Fisch.) Bge. or Astragali Radix membranaceus (Fisch.) Bge. var. mongholicus (Bge) Hsiao, is a commonly used traditional Chinese medicine for the treatment of liver diseases. This study aimed to comprehensively evaluate the pharmacological action and explore the potential mechanism of AR on liver fibrosis. Rats were administered with carbon tetrachloride for eight weeks, followed by oral treatment with AR for six weeks. The efficacy was confirmed by measuring liver function and liver fibrosis levels. The underlying mechanisms were explored by detecting the expression of related proteins. AR significantly decreased the serum levels of alanine aminotransferase (ALT), aspartate aminotransferase (AST), collagen IV (COL-IV), hyaluronic acid (HA), laminin (LN), and precollagen type III (PCIII). In addition, AR inhibited the deposition of collagen and the activation of hepatic stellate cells. Those data strongly demonstrated that AR alleviated liver fibrosis by $\mathrm{CCl}_{4}$. In order to illustrate the potential inflammatory, the mRNA levels of IL- 6 , TNF- $\alpha$, and IL- $1 \beta$ were detected. Subsequently, immunohistochemistry analysis was performed to further verify the expression of type I collagen. Finally, the expression of key proteins in the inflammatory signaling pathway was detected. AR significantly reduced the expression of highmobility group box 1 (HMGB1), TLR4, Myd88, RAGE, and NF- $\kappa$ B p65 genes and proteins. In addition, western blotting showed AR decreased the protein expression of RAGE, p-MEK1/2, p-ERK1/2, and p-c-Jun. Taken together, our data reveal that AR significantly inhibits liver fibrosis by intervening in the HMGB1-mediated inflammatory signaling pathway and secretion signaling pathway. This study will provide valuable references for the in-depth research and development of Astragali Radix against liver fibrosis.

\section{Introduction}

Liver fibrosis is a complex pathological process and an intermediate link between various liver diseases including cirrhosis and liver cancer $[1,2]$. And prevention of malignant liver diseases by inhibiting the occurrence and development of liver fibrosis has attracted worldwide attention [3]. Numerous studies have revealed the pathogenesis of liver fibrosis, such as inflammatory response, activation of hepatic stellate cells, and excessive deposition of extracellular matrix, but it has not yet been transformed into an effective and powerful treatment $[4,5]$. There is still a lack of safe and effective means of treating liver fibrosis $[6,7]$.

It is necessary to develop new drugs for treating liver fibrosis [7]. Traditional Chinese medicine has potential advantages in the treatment of chronic diseases, so we tried to find effective drugs for the treatment of liver fibrosis. Astragali Radix (AR), the dried root of Astragali Radix membranaceus (Fisch.) Bge. or Astragali Radix membranaceus (Fisch.) Bge. var. mongholicus (Bge) Hsiao, is a 
commonly used traditional Chinese medicine for the treatment of liver diseases [8]. And it has been proved that AR possesses hepatoprotective and anti-inflammatory properties and has been shown to exhibit immunomodulating and antioxidant activity, among others [9]. In traditional Chinese formula, AR is often used for the treatment of liver fibrosis [10]. Although the protective effects of AR on liver injury and related mechanisms have been reported $[9,11]$, the studies on the effect and mechanism of AR on liver fibrosis are still lacking. More importantly, toxicological study has confirmed the high safety of AR. No significant toxicity or side effects were observed when the dose administered to rats was set to $39.9 \mathrm{~g} / \mathrm{kg}$ [12].

Experimental and clinical studies have found that the high-mobility group box-1 (HMGB1), recently discovered damage-associated molecular patterns (DAMPs), is substantially enriched in patients with hepatic fibrosis $[13,14]$. HMGB1 is a late inflammatory mediator that plays an important role in the pathogenesis of many chronic inflammations, which is the main cause of liver fibrosis [15]. There is evidence that HMGB1 can directly stimulate hepatic stellate cells (HSCs) activation to accelerate the development of liver fibrosis [16]. More importantly, toll-like receptor 4 (TLR4) and the receptor of advanced glycation end products (RAGE) are the main receptors of HMGB1 [15]. It is reported that the binding of extracellular HMGB1 to TLR4 could activate NF- $\kappa \mathrm{B}$ signaling to induce inflammation and aggravate liver fibrosis. Studies have shown that extracellular HMGB1 binding to RAGE could phosphorylate c-Jun to induce a significant increase in type I collagen secretion $[16,17]$, which directly accelerates the development of liver fibrosis (c-Jun is a component of the heterodimeric AP1 and is a potent transactivating factor for the collagen $1 a 1$ and collagen $1 a 2$ genes $[17,18])$.

Based on previous studies, this study aims to provide answers to the following questions: Does AR have therapeutic effect on carbon tetrachloride- $\left(\mathrm{CCl}_{4^{-}}\right)$induced liver fibrosis in rats? What is the underlying mechanism? Is the mechanism related to downregulation of HMGB1?

\section{Materials and Methods}

2.1. Reagents. In this study, colchicine was purchased from Xishuangbanna Banna Pharmaceutical Co. Ltd. (Yunnan, China). $\mathrm{CCl}_{4}$ was supplied by Tianjin Guangfu Chemical Research Institute (Tianjin, China). Alanine amino transferase (ALT, Cat. No.: 20180627), aspartate amino transferase (AST, Cat. No.: 20180607), collagen IV (COL-IV, Cat. No.: 201809), hyaluronic acid (HA, Cat. No.: 201808), laminin (LN, Cat. No.: 201809), and precollagen type III (PC III, Cat. No.: 201809) detection kits were provided by Nanjing Jiancheng Bioengineering Institute (Nanjing, China). The antibodies of phospho-c-Jun, phospho-MEK1/2, HMGB1, $\alpha$-SMA, phospho-ERK1/2, and ERK1/2 were bought from Cell Signaling Technology (United States). The antibodies of MEK1/2 and COL-I were purchased from Abcam (United States) and the antibodies of RAGE and GAPDH were bought from ABclonal (China).
2.2. Plant Material. AR (the dried root of $A R$ membranaceus (Fisch.) Bge.) was supplied by Beijing Lvye Pharmaceutical Co. Ltd. (Beijing, China). Ten times of water was used for the first extraction of AR for $2 \mathrm{~h}$. Then, eight times of water was used to extract for 1.5 hours. The extract of Astragalus membranaceus was dried into powder by freeze vacuum drying oven. The final yield was about $40.08 \%$. The Q-TOF LC/MS analysis showed that the main components of AR extract included 3,4-dihydroxybenzyl aldehyde (6.17\%), calycosin-7-glucoside (0.69\%), armillaripin (10.31\%), rhodomollein III (7.05\%), phthalic anhydride $(1.76 \%)$, clavatine $(6.46 \%)$, pterosin B (7.46\%), D-cathinone (2.65\%), formononetin $(15.4 \%)$, Andrographolide $(2.57 \%)$, and anemarrhenasaponin-I (4.55\%) [19].

2.3. Animals and Administration. A total of 36 male Sprague Dawley rats weighting 180-200 g were obtained from the China Food and Drug Certification Research Institute (Permission No. SCXK (Jing)-2014-0013). Those rats were housed in the Central Animal Laboratory of the Fifth Medical Center of Chinese PLA General Hospital. The animals were maintained under controlled conditions of temperature $25^{\circ} \mathrm{C} \pm 2{ }^{\circ} \mathrm{C}$, humidity $55 \% \pm 5 \%$, and with light and dark cycles.

All the animals were randomly divided into six groups with six rats in each group. Olive oil diluted $\mathrm{CCl}_{4}$ in a ratio of $1: 1$. The model group, AR $(2.7,5.4,10.8 \mathrm{~g} / \mathrm{kg} / \mathrm{d})$ groups, and colchicine (as a positive control group) groups were given $\mathrm{CCl}_{4}$ diluent $(2 \mathrm{~mL} / \mathrm{kg})$ twice a week for 8 weeks [19]. At the same time, the control group was intraperitoneally injected with the same dose of olive oil. Eight weeks later, the AR (2.7, 5.4, and $10.8 \mathrm{~g} / \mathrm{kg} / \mathrm{d})$ groups and colchicine group $(0.2 \mathrm{mg} / \mathrm{kg} / \mathrm{d})$ were given the corresponding concentration of drugs [20]. The control group and model group were given equal volume of distilled water. All rats were treated by intragastric administration once a day for 6 weeks. All rats were deprived of food for $24 \mathrm{~h}$ before the last treatment but allowed free access to water. The animals were sacrificed $2 \mathrm{~h}$ after the last treatment and their liver and serum were harvested for the further studies. The blood samples were centrifuged at $3500 \mathrm{rpm}$ for $15 \mathrm{~min}$. The liver tissue was divided into 3 pieces and placed in the sample bottle. Serum and liver tissue were stored at $-80^{\circ} \mathrm{C}$. All animal experiments were approved by the Ethical Committee of the Fifth Medical Center of Chinese PLA General Hospital in China.

2.4. Biochemical Assay. The serum levels of ALT, AST, COLIV, HA, LN, and PC III were measured. In brief, according to the manufacturer's protocols, the samples, 2,4-dinitrophenylhydrazine solution, and sodium hydroxide solution were added in turn. The final color was read at $510 \mathrm{~nm}$, and the serum ALT and AST levels were determined according to the OD value. Similarly, according to the manufacturer's instructions, add the reagents in the kit in turn, read the final color at $450 \mathrm{~nm}$, and obtain the serum 
levels of COL-IV, HA, LN, and PC III according to the OD value.

2.5. Histological Examination. Liver tissue was fixed in 10\% neutral buffered formalin for more than $48 \mathrm{~h}$. All the livers were eluted with gradient ethanol solution and fixed and embedded in paraffin. Tissue sections about $4-5 \mu \mathrm{m}$ in thickness were cut and stained with hematoxylin eosin (H\&E) and Masson. The pathological changes in the liver tissues were observed under a Nikon microscope (Nikon Instruments Inc., Japan).

2.6. Quantitative Reverse Transcription Polymerase Chain Reaction (RT-PCR) Analyses. Quantitative RT-PCR was used to detect the mRNA expression of IL- 6 , TNF- $\alpha$, IL- $1 \beta$, HMGB1, TLR4, Myd88, and RAGE in liver tissues. Total RNA was extracted from $90 \mathrm{mg}$ frozen liver tissue by Trizol reagent (Life Technologies, CA, USA). The absorbance of the extracted mRNA was detected by spectrophotometer at $260 \mathrm{~nm}$ and $280 \mathrm{~nm}$, respectively. The mRNA with purity (A260/A280) between 1.8 and 2.2 was selected as the qualified product for the follow-up study. RNA $(2 \mu \mathrm{g})$ was transcribed into cDNA by PrimerScript RT regent kit (Thermo Fisher Scientific, MA, USA). The cDNA was subsequently subjected to PCR amplification by ABI 7500 Real-Time PCR machine (Applied Biosystems Inc, Carlsbad, CA, USA). Data analysis was performed by the $2^{-\triangle \Delta C T}$ method. The primers are listed in Table 1.

2.7. Western Blotting. The mixture of ice-cold radio immunoprecipitation assay (RIPA) lysis buffer, phenylmethylsulfonyl fluoride (PMSF), and protein phosphatase inhibitor was used for protein extraction. Liver tissue $(80 \mathrm{mg})$ was homogenized and lysed in the mixture, and then centrifuged at $12000 \times \mathrm{g}$ and $4^{\circ} \mathrm{C}$ for $10 \mathrm{~min}$. The supernatant was collected and BCA Protein Assay kit (Beijing Solarbio Science \& Technology Co., Ltd. Beijing, China) was used to detect the total protein concentration. All protein samples were levelled to the same concentration. Then, reducing Laemmli SDS sample buffer was added and mixed well, and boiled in boiling water for 5 minutes. The prepared samples were western blotted with $10 \%$ sodium dodecyl sulfatepolyacrylamide gel electrophoresis (SDS-PAGE) and the blot was transferred to the polyvinylidene fluoride (PVDF) membrane. The PVDF membrane was sealed in 5\% skimmed milk at room temperature for 2 hours and then placed in a mixture of 5\% skimmed milk, Tris-buffered saline (TBS), and $0.05 \%$ Tween 20 with the corresponding primary antibody, and incubated overnight at $4^{\circ} \mathrm{C}$. Antibodies, including rabbit Anti-NF- $\kappa$ B p65 (Cell Signaling Technology, dilution: 1: 1000), RAGE (ABclonal technology, dilution: 1:1000), phospho-MEK1/2 (Cell Signaling Technology, dilution: 1: 2000), MEK1/2 (Abcam, dilution: $1: 10000$ ), phospho-ERK1/ 2 (Cell Signaling Technology, dilution: $1: 1000$ ), ERK1/2 (Cell Signaling Technology, dilution: 1:10000), phospho-c-Jun (Abcam, dilution: 1:500), and GAPDH (ABclonal technology, dilution: $1: 10000$ ), were used at this step. GAPDH was used as control for total protein extract. After incubation with appropriate secondary antibody for 2 hours at room temperature, the membrane was washed 3 times in TBS with $0.05 \%$ Tween 20 for 5 minutes each. Finally, the chemiluminescence system was used to measure immunoreactive proteins. All the western blotting was repeated 3 times.

2.8. Immunohistochemistry Analysis. To evaluate the effects of AR on the expression of $\alpha$-SMA, HMGB1, and collagen I in liver tissue of fibrosis rats, immunohistochemical analysis was performed as described previously [21]. Paraffin sections of liver tissues were put in xylene and gradient ethanol for deparaffinization and dehydration, and then the sections were put into citric acid antigen retrieval solution for antigen retrieval. The sections were treated with 3\% hydrogen peroxide solution to block endogenous peroxidase. The sections were then blocked with $1 \%$ BSA for 30 minutes at room temperature. Subsequently, the sections were incubated with $\alpha$-SMA (Cell Signaling Technology, dilution: 1:100), Rabbit AntiHMGB1 (Cell Signaling Technology, dilution: 1:200), and collagen type I (Abcam, dilution: $1: 200$ ) in a PBS solution at $4^{\circ} \mathrm{C}$ overnight. After incubating the sections with appropriate secondary antibodies for 1 hour at room temperature, they were stained with DAB staining solution and hematoxylin, and finally the slides were dehydrated and mounted. Nikon microscope (Nikon Instruments Inc., Japan) and NIS-Elements (F 4.0 version, Japan) software were used to observe the sections. Finally, Image-Pro Plus (version 6.0, Media Cybemetics, INC., Rockville, MD, USA) software was used to calculate and analyze the average integrated optical density (IOD) of the positive area of the immunohistochemical reaction image.

2.9. Statistical Analysis. Results were expressed as mean\pm standard deviation (SD). Data were evaluated by one-way ANOVA and Duncan's multirange test with the SPSS computer program (version 20.0, SPSS Inc., Chicago, IL, USA). $P<0.05$ was considered statistically significant and $P<0.01$ was considered highly significant.

\section{Results}

3.1. AR Has Therapeutic Effect on $\mathrm{CCl}_{4}$-Induced Liver Injury. As shown in Figures 1(a) and 1(b), the levels of ALT and AST in rats in the model group were significantly increased $(P<0.01)$ compared with those in the control group. And serum ALT and AST concentrations were significantly reduced after oral administration of AR $5.4 \mathrm{~g} / \mathrm{kg}$ and $10.8 \mathrm{~g} / \mathrm{kg}$ $(P<0.01)$. Subsequently, the serum levels of COL-IV, HA, LN, and PC III which could reflect the degree of hepatic fibrosis were examined [22]. As shown in Figures 1(c)-1(f), the serum levels of COL-IV, HA, LN, and PC III in the model group were significantly higher than those in the control group $(P<0.01)$. The inhibitory effect of AR on the levels of COL-IV, HA, LN, and PC III is dose-dependent. In particular, the serum levels of COL-IV, HA, LN, and PC III were significantly decreased in the $10.8 \mathrm{~g} / \mathrm{kg}$ AR group $(P<0.01)$. 
TABLe 1: Primers sequences for RT-PCR.

\begin{tabular}{lcc}
\hline Gene & Sense primer $\left(5^{\prime}-3^{\prime}\right)$ & Antisense primer $\left(5^{\prime}-3^{\prime}\right)$ \\
\hline IL-6 & AGGAGTGGCTAAGGACCAAGACC & TGCCGAGTAGACCTCATAGTGACC \\
TNF- $\alpha$ & GCATGATCCGAGATGTGGAACTGG & CGCCACGAGCAGGAATGAGAAG \\
IL-1 $\beta$ & ATCTCACAGCAGCATCTCGACAAG & CACACTAGCAGGTCGTCATCATCC \\
HMGB1 & ACAACACTGCTGCGGATGACAAG & CCTCCTCGTCGTCTTCCTCTTCC \\
TLR4 & GCTGCCAACATCATCCAGGAAGG & TGATGCCAGAGCGGCTACTCAG \\
Myd88 & CGACGCCTTCATCTGCTACTGC & CCACCACCATGCGACGACAC \\
RAGE & CTGCCTCTGAACTCACAGCCAATG & GTGCCTCCTGGTCTCCTCCTTC \\
GAPDH & GTCCATGCCATCACTGCCACTC & GATGACCTTGCCCACAGCCTTG \\
\hline
\end{tabular}

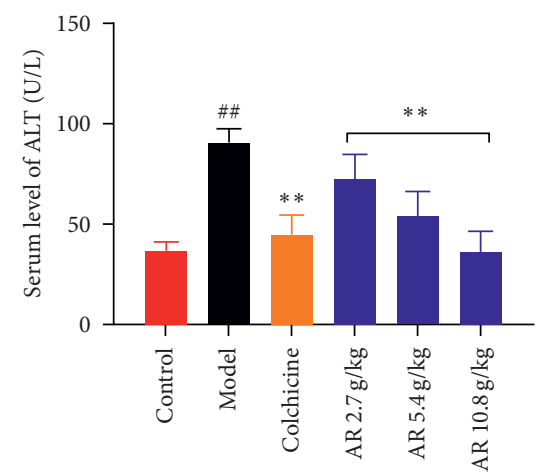

(a)

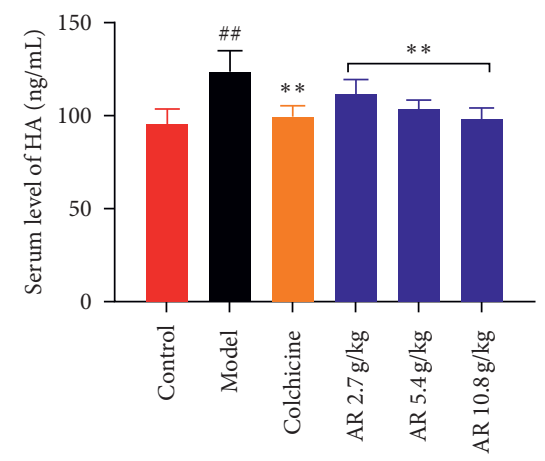

(d)

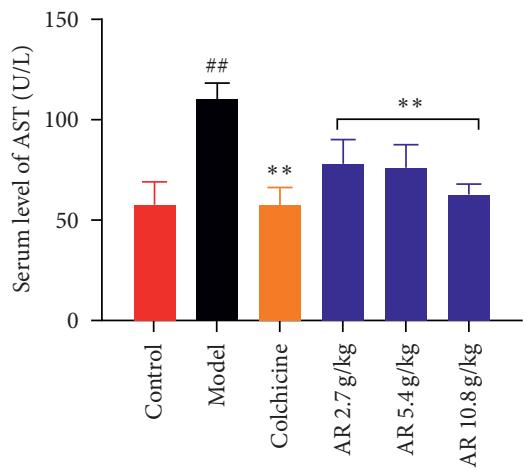

(b)

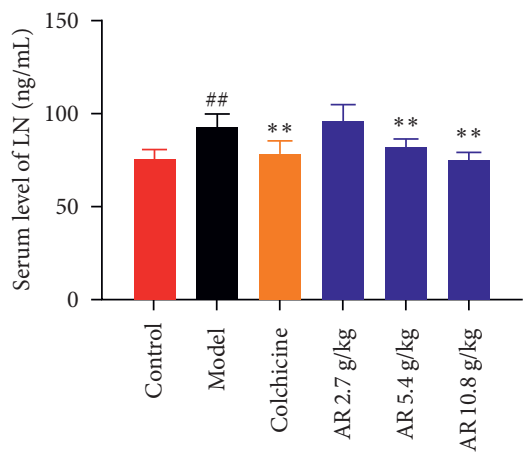

(e)

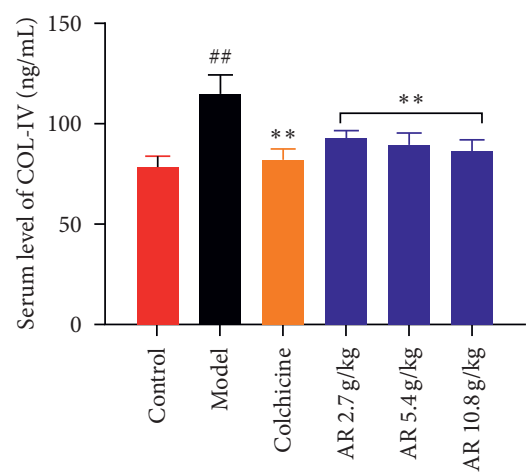

(c)

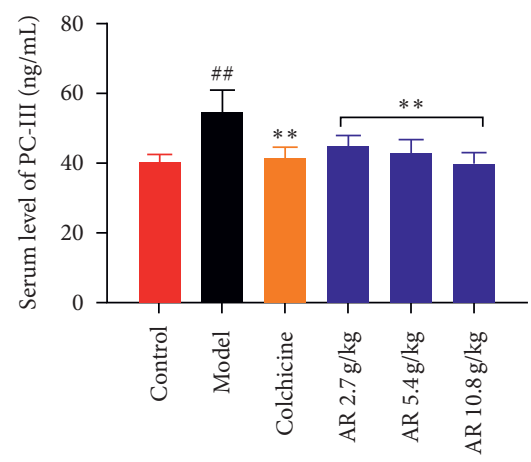

(f)

FIgURE 1: Effect of AR on $\mathrm{CCl}_{4}$-induced liver fibrosis in rats in different groups. (a) Serum level of ALT, (b) serum level of AST, (c) serum level of COL-IV, (d) serum level of HA, (e) serum level of LN, and (f) serum level of PC-III. Values are expressed as mean \pm SD $(n=6)$. ${ }^{\#} P<0.05$ and ${ }^{\# \#} P<0.01$ when compared with the control group. ${ }^{*} P<0.05$ and ${ }^{* *} P<0.01$ when compared with the model group.

\subsection{The Effect of AR on Liver Histopathological Changes and} Liver Fibrosis. Then, hematoxylin-eosin (HE) staining and Masson staining were used to observe the pathological changes of liver tissue $[21,23]$. The results of $\mathrm{HE}$ showed that the liver tissue structure of the control group was normal without morphological changes. The long-term $\mathrm{CCl}_{4}$-treated rats showed partial necrotic in their liver accompanied by severe steatosis and inflammatory cell infiltration around the central vein of hepatic lobules. In contrast, the degree of liver damage, steatosis, and inflammatory infiltration in the AR treatment groups were reduced to varied degrees in a dosedependent manner (Figure 2(a)). In the liver section of Masson staining, the blue area represented the deposited collagen. The results show that a large amount of collagen was deposited around the hepatic sinus of the model group. AR can effectively reduce collagen deposition and improve liver fibrosis (Figure 2(b)). The above results confirmed that the $\mathrm{CCl}_{4}$-induced rat liver fibrosis model was successful, and AR could effectively ameliorate liver fibrosis in rats.

3.3. Inflammatory Response in Rats Induced by $\mathrm{CCl}_{4}$. Clinical data suggest that inflammation is a key factor in the progression of liver fibrosis [24]. IL-6, as a classic proinflammatory cytokine biomarker, is used for clinical diagnosis of chronic liver fibrosis [25]. TNF- $\alpha$ can promote the survival of activated HSCs and promote the development of liver fibrosis [26]. It is reported that the serum IL-1 $\beta$ in patients with liver fibrosis caused by schistosomiasis is significantly increased [24]. Therefore, we detected the expression of IL-6, TNF- $\alpha$, and IL- $1 \beta$ in rats by quantitative RT-PCR. The mRNA levels of IL-6, TNF- $\alpha$, and IL- $1 \beta$ were increased remarkably in 


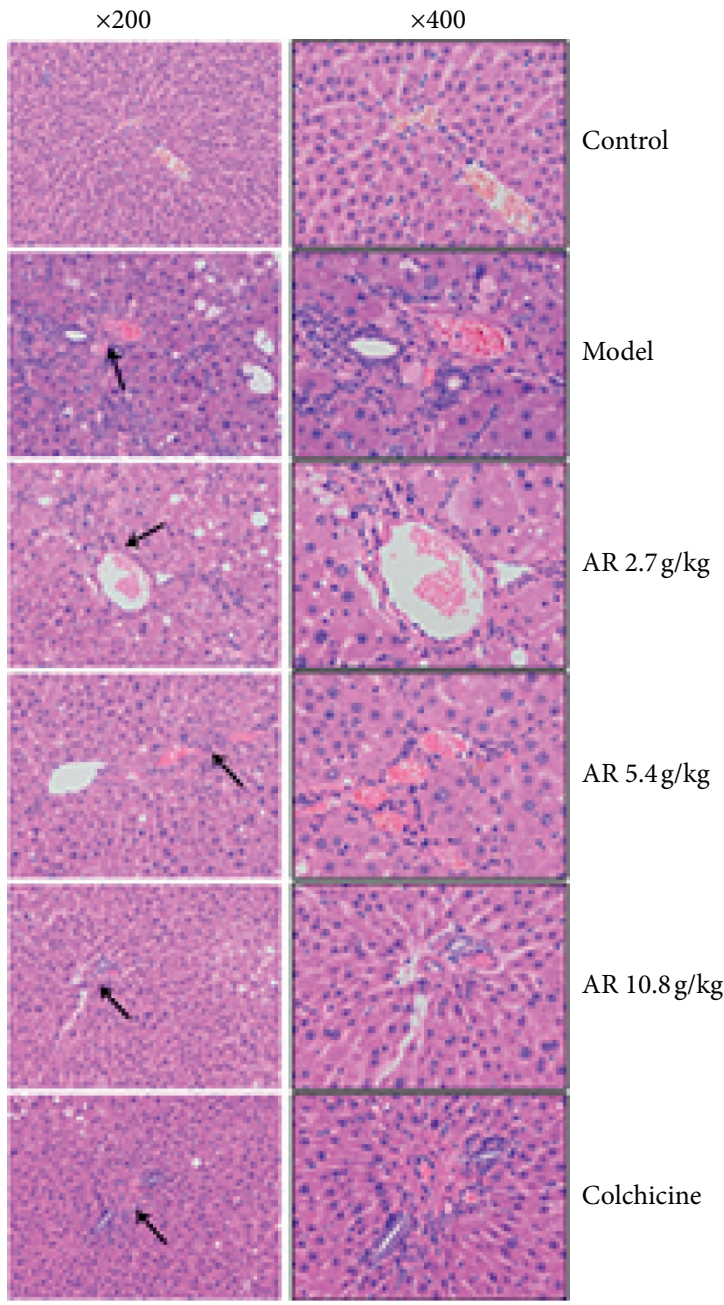

(a)
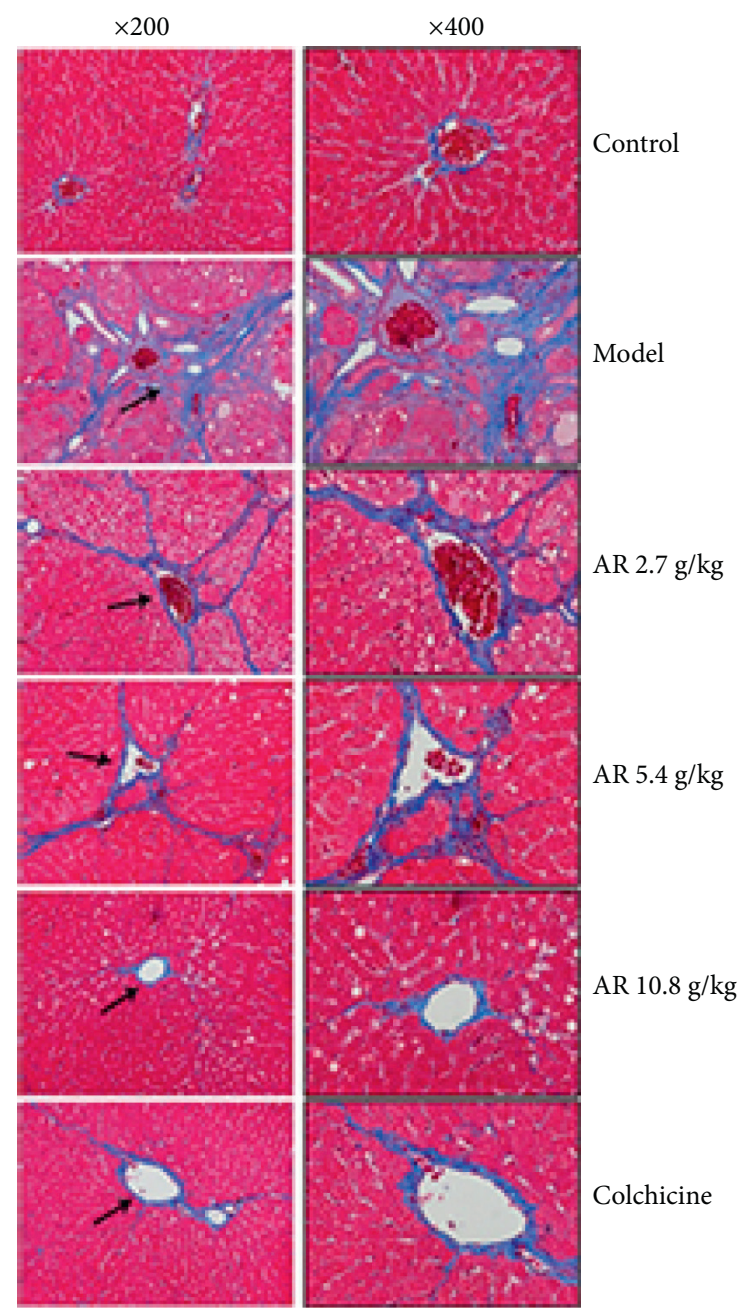

(b)

Figure 2: Histopathological observation of AR on improvement of liver fibrosis in rats. (a) Hematoxylin and eosin- (HE-) stained liver section in six groups (original magnification, $\times 200, \times 400$ ). (b) Histological examination of liver section with Masson stain (original magnification, $\times 200, \times 400$ ). Blue areas show collagen fibers and damaged liver tissue.

the $\mathrm{CCl}_{4}$-induced group compared with the control group $(P<0.01$, Figure 3$)$. After treatment with $\mathrm{AR}$, the expression of related genes decreased in a dose-dependent manner $(P<0.05, P<0.01)$. The results showed that AR had therapeutic effect on $\mathrm{CCl}_{4}$-induced liver inflammation in rats.

\subsection{AR Downregulates $\mathrm{CCl}_{4}$-Induced Liver Inflammation.} Literature research found that HMGB1, a cytokine abundantly enriched in patients with liver fibrosis, mediated TLR4//NF- $\kappa \mathrm{B}$ signaling pathway is one of the important pathways for liver aseptic inflammation. Therefore, we tested the expression levels of HMGB1, TLR4, Myd88, and NF- $\kappa \mathrm{B}$ p65, which are the key targets of this signaling pathway in liver fibrosis rats. Quantitative RT-PCR and immunohistochemistry (IHC) methods were used to detect the expression and subcellular localization of HMGB1. As shown in Figures 4(a)-4(e), in the control group, HMGB1 was mainly located in the nucleus of hepatocytes and rarely in the cytoplasm or sinusoids. In the model group, the greater HMGB1 immunoreactivity was observed in the periportal and bridge fibrosis areas in the liver. In addition, computer-assisted semiquantitative analysis showed that AR treatment alleviated the expression of HMGB1 compared with the model group (Figure 4(f), $P<0.05$, $P<0.01)$.

Furthermore, the mRNA expression of HMGB1 was also quantified by using the RT-PCR method. The results showed that high dose of AR could significantly reduce the mRNA expression of HMGB1, which is consistent with the result of the IHC method. Those results proved that AR could reduce the expression of HMGB1 (Figure 4(g)). Subsequently, quantitative RT-PCR was used to detect the TLR4, Myd88, and RAGE mRNA levels in the model group, which were significantly higher than those in the control group (Figures $4(\mathrm{~h})-4(\mathrm{j}), P<0.01)$. After treatment with AR, the expression of related genes decreased in a dose-dependent manner $(P<0.05, P<0.01)$. Western blotting was used to 


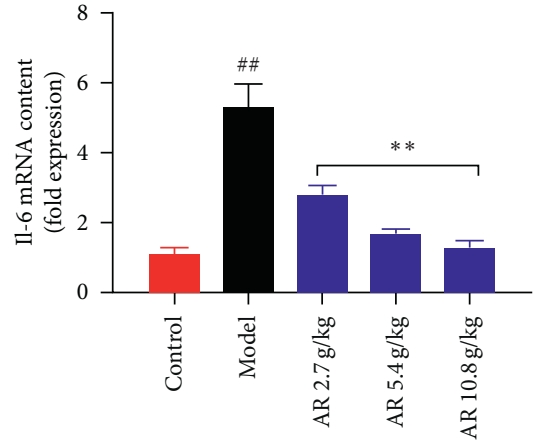

(a)

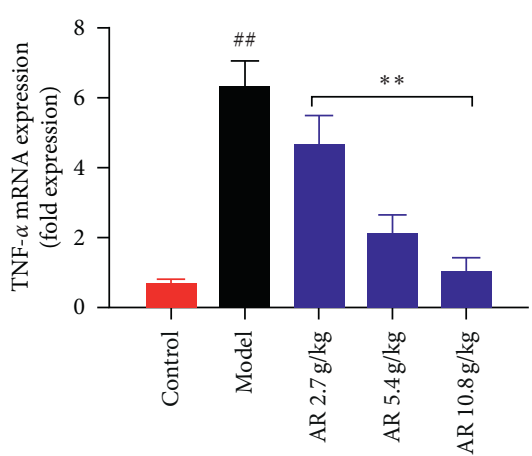

(b)

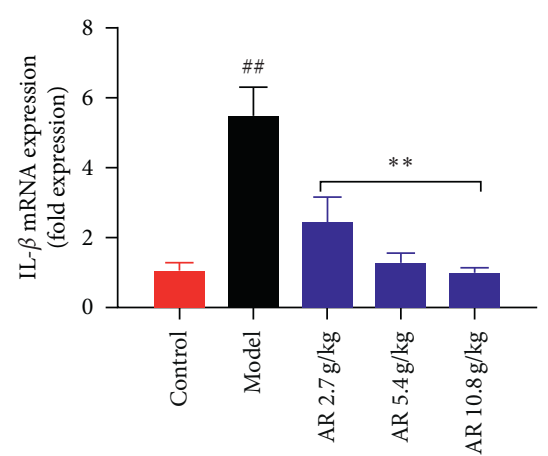

(c)

FIGURE 3: Effect of AR on inflammatory factors levels in liver fibrosis induced by CCl 4 . (a) IL-6 mRNA level. (b) TNF- $\alpha$ mRNA level. (c) IL$1 \beta$ mRNA level. Values are expressed as mean \pm SD $(n=6) .{ }^{\#} P<0.05$ and ${ }^{\# \#} P<0.01$ when compared with the control group. ${ }^{*} P<0.05$ and ${ }^{* *} P<0.01$ when compared with the model group.

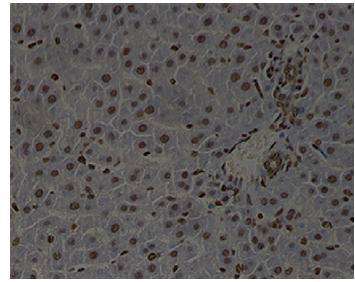

(a)

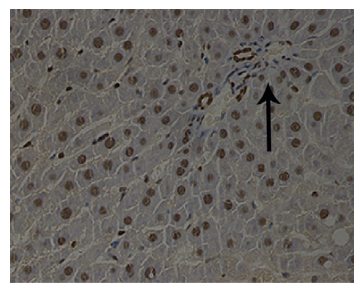

(e)

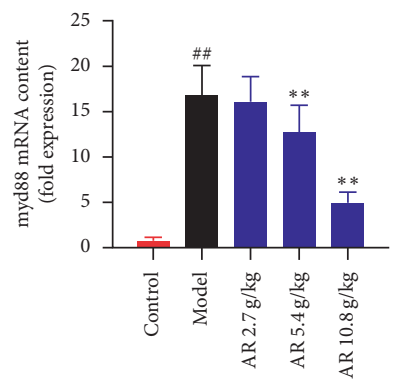

(i)

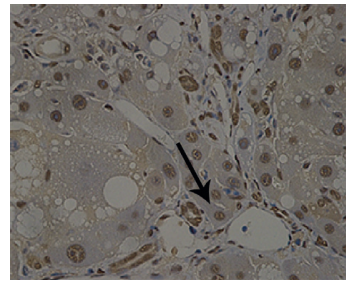

(b)

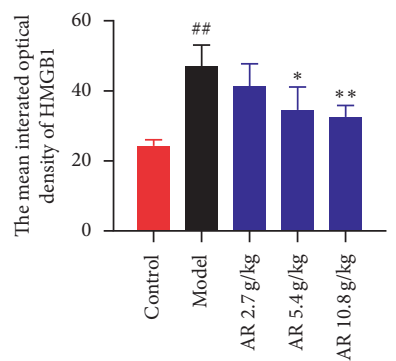

(f)

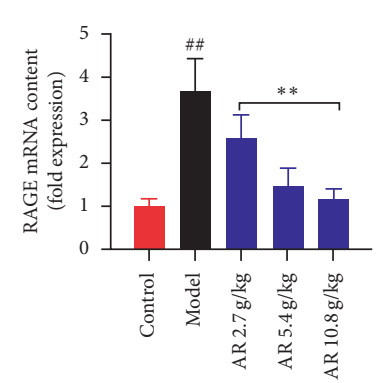

(j)

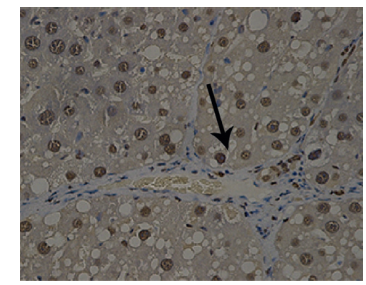

(c)

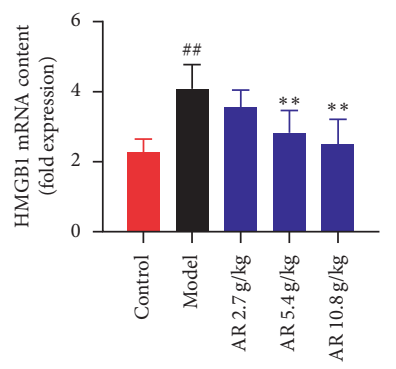

(g)

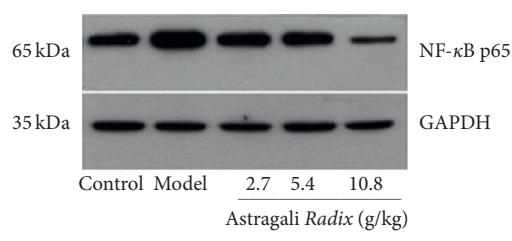

(k)

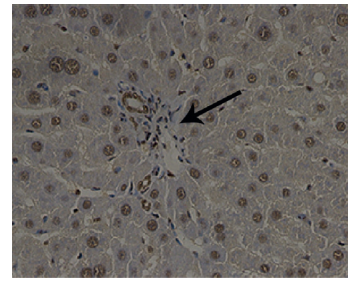

(d)

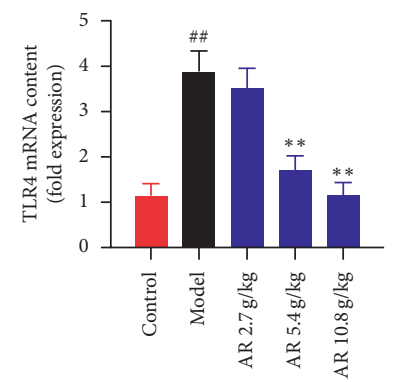

(h)

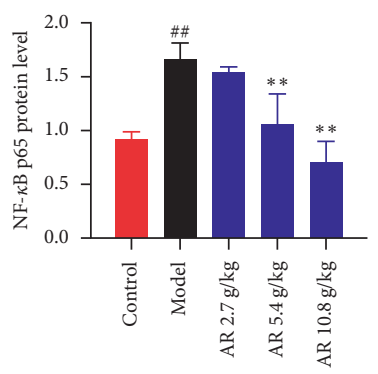

(1)

FIGURE 4: Effect of AR on HMGB1/TLR4/NF- $\kappa$ B signaling pathway in liver fibrosis induced by $\mathrm{CCl}_{4}$. (a-e) Immunohistochemistry analysis for the effect of AR on HMGB1 localization (original magnification, $\times 400 ; n=3$; (a) control group; (b) model group; (c) $2.7 \mathrm{~g} / \mathrm{kg}$ AR group; (d) $5.4 \mathrm{~g} / \mathrm{kg}$ AR group; (e) $10.8 \mathrm{~g} / \mathrm{kg}$ AR group). (f) The mean integrated optical density of HMGB1. (g) HMGB1 mRNA level. (h) TLR4 mRNA level. (i) Myd88 mRNA level. (j) RAGE mRNA level. (k) Western blot images of NF- $\kappa$ B p65. (l) NF- $\kappa$ B p65 protein level. Values are expressed as mean $\pm \mathrm{SD}(n=6) .{ }^{\#} P<0.05$ and ${ }^{\# \#} P<0.01$ when compared with the control group. ${ }^{*} P<0.05$ and ${ }^{* *} P<0.01$ when compared with the model group.

detect the expression level of NF- $\kappa \mathrm{B}$ p65 in the liver of rats with liver fibrosis. As shown in Figures $4(\mathrm{k})-4(\mathrm{l})$, the immunoblotting band of NF- $\kappa \mathrm{B}$ p65 in the model group was significantly thicker than that of the control group, and the statistical results showed significant differences $(P<0.01)$. After AR treatment, the expression of NF- $\kappa$ B p 65 decreased in 
a dose-dependent manner, especially in the $5.4 \mathrm{~g} / \mathrm{kg}$ AR group and $10.8 \mathrm{~g} / \mathrm{kg}$ AR group $(P<0.01)$. These results suggest that AR may improve liver inflammation by downregulating HMGB1/TLR4//NF- $\kappa$ B signaling pathway.

3.5. AR Inhibits the Activation of HSCs. HSCs are the core cells of liver fibrosis. It is reported that HMGB1 can directly activate HSCs [16]. Therefore, we speculate that AR can play a protective role in liver fibrosis by reducing the activation of HSCs. $\alpha$-smooth muscle actin ( $\alpha$-SMA) is a special marker of activated HSCs [27]. Here, the ratio of activated HSCs was measured by using IHC method through detecting $\alpha$-SMA in the liver tissues (Figure 5). In the control group, only a small amount of $\alpha$-SMA-positive cells was found in the portal area, which might be the hepatic arteries. More $\alpha$-SMA-positive cells were observed in the $\mathrm{CCl}_{4}$-induced group compared with control group, and the $\alpha$-SMA-positive cells were significantly reduced after AR treatment. By computer-assisted semiquantitative analysis, the mean integrated optical density (IOD) of the $\alpha$-SMA-positive area of the AR treatment group was confirmed to be significantly reduced in a dose-dependent manner compared to model group (Figure 5, $P<0.01$ ). Those results revealed that $\mathrm{AR}$ could reduce the activation of HSCs, a key cell of liver fibrosis.

3.6. AR Has an Effect on the Type 1 Collagen Secretion Signaling Pathway Mediated by HMGB1. Next, IHC method was used to detect the expression of collagen type I. In Figures 6(a)6(e), type I collagen deposition around the hepatic lobular portal vein in rats treated with $\mathrm{CCl}_{4}$ alone was significantly increased. After treatment with $\mathrm{AR}$, the expression and deposition of type I collagen in the liver were significantly inhibited, especially in the group with the dose of $10.8 \mathrm{~g} / \mathrm{kg}$. The semiquantitative analysis of type I collagen immunopositive region further confirmed the above results (Figure 6(f)). The mean IOD of type I collagen in the AR administered group was significantly reduced in a dosedependent manner compared to the model group $(P<0.01)$. It was previously reported that HMGB1 interacted with the receptor of RAGE to regulate the expression of type I collagen [17]. Therefore, we explored the effects of AR on the expression of downstream targets of HMGB1, such as RAGE, p-MEK1/2, p-ERK1/2, p-cJun, and type I collagen. Western blot method was used to detect the expression of RAGE, p-MEK1/2, p-ERK1/2, and p-cJun protein after administration of AR (Figures $6(\mathrm{~g})-6(\mathrm{k})$ ). The proteins of RAGE, p-MEK1/2, p-ERK1/2, and p-cJun were increased remarkably only in the $\mathrm{CCl}_{4}$-induced group compared with the control group $(P<0.05, P<0.01)$. Compared with the model group, the high-dose administration group of $A R$ significantly reduced the expression of RAGE, $\mathrm{p}-\mathrm{MEK} 1 / 2$, $\mathrm{p}$-ERK1/2, and p-cJun (Figures 6(h)-6(k), $P<0.05$, $P<0.01)$.

\section{Discussion}

In this study, the aqueous extract of AR was used, which is consistent with the clinical use of traditional Chinese medicine. And the research results are more valuable. To determine whether AR has a protective effect on liver fibrosis, $\mathrm{CCl}_{4}$-induced liver fibrosis model was performed in rats. Consistent with clinical observations, AR could improve liver function to some extent to alleviate liver injury $[28,29]$. In this study, the results demonstrated that AR could reduce serum markers of liver fibrosis, including COLIV, HA, LN, and PC-III. The examination of serum levels and liver histopathology exhibited that AR showed obvious anti-liver fibrosis effect and the anti-liver fibrosis effect of AR at the dose of $10.8 \mathrm{~g} / \mathrm{kg}$ is comparable to that of colchicine. In order to apply AR more scientifically, the potential mechanism of AR on alleviating liver fibrosis was further explored.

Previous reports and the results of this study confirmed that AR has definite hepatoprotective effect and can effectively improve liver fibrosis [19, 30, 31]. However, how to exert the therapeutic effect is still unclear. Previous studies have reported that AR has strong anti-inflammatory activity and can effectively reduce the expression of inflammatory factors such as iNOS, cyclooxygenase-2 (COX-2), IL-6, TNF- $\alpha$, and IL- $\beta[32,33]$. Long-term inflammatory response is the key to the occurrence of liver fibrosis [34]. Therefore, we detected the effect of AR on the expression of inflammatory factors in liver fibrosis rats. The results showed that AR could significantly downregulate the expression of IL- $1 \beta$, TNF- $\alpha$, and IL- 6 . It suggested that AR might improve liver fibrosis by inhibiting the inflammatory response in rats.

Pathogen-free inflammatory liver disease is considered "sterile" [35]. Studies have reported that DAMP is the key to aseptic inflammation after liver injury, and HMGB1 is the key point [36]. Extracellular HMGB1 is a multifunctional cytokine, which induces inflammation by binding with specific cell surface receptor TLR4 [37]. Thus, we detected the expression of HMGB1 in rats to explore whether AR had effect on regulating HMGB1 expression via improving the inflammatory response. The results showed that the expression of HMGB1 decreased in the AR treatment group compared with the model group. The TLR4 signaling pathway is the most studied pathway. TLR4, as a pattern recognition receptor, recruits Myd88 under the action of the endogenous activator HMGB1, which causes the activation of the NF- $\kappa$ B inflammatory pathway and promotes the transcription of inflammatory cytokines IL- $1 \beta$, TNF- $\alpha$, and IL-6 $[38,39]$. Therefore, we detected the expression of TLR4, Myd88, and NF- $\kappa \mathrm{B}$ p65. The results showed that the expression levels of TLR4, Myd88, and NF- $\kappa$ B p65 in AR treatment group were significantly lower than those in the model group. The above results suggest that AR might reduce $\mathrm{CCl}_{4}$-induced liver inflammatory by downregulating the expression of $\mathrm{HMGB} 1 / \mathrm{TLR} 4 / \mathrm{NF}-\kappa \mathrm{B}$ signaling pathway.

In experimental and human liver injury, activated HSCs (myofibroblasts) are recognized as the driving factors of liver fibrosis [40]. It has been reported that HSCs activated by inflammatory factors are the main source of myofibroblasts in the liver [34]. And HMGB1 could directly stimulate the activation of HSCs [16]. The pharmacodynamic results show that $\mathrm{AR}$ can effectively improve the liver fibrosis in rats induced by $\mathrm{CCl}_{4}$. The study also found that AR can not only 


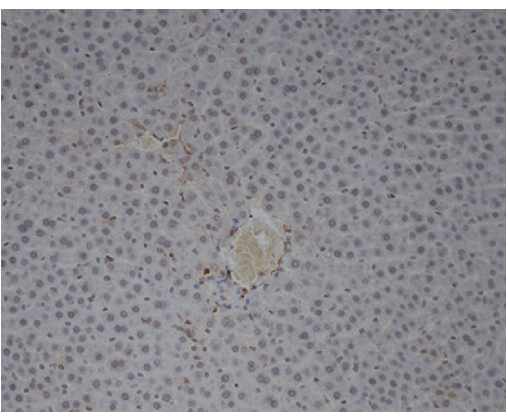

(a)

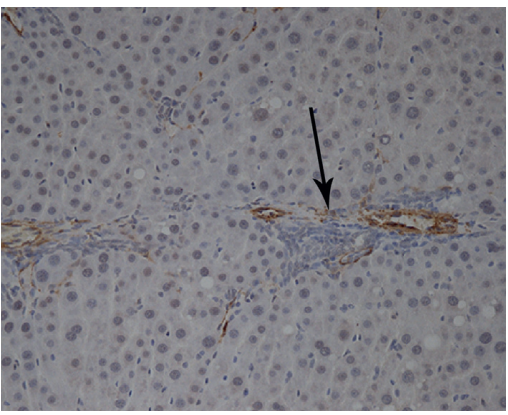

(d)

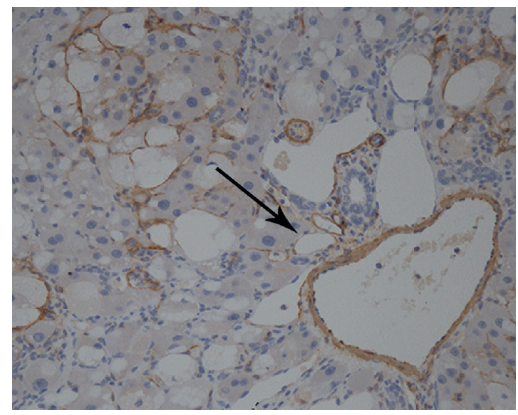

(b)

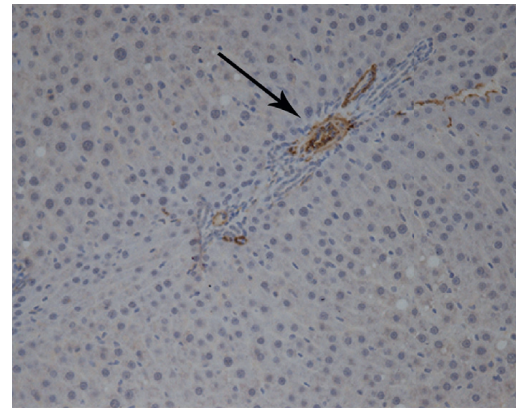

(e)

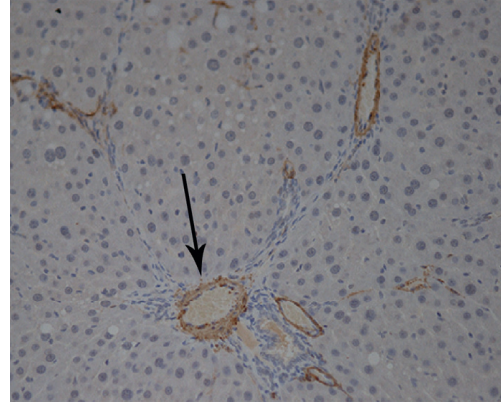

(c)

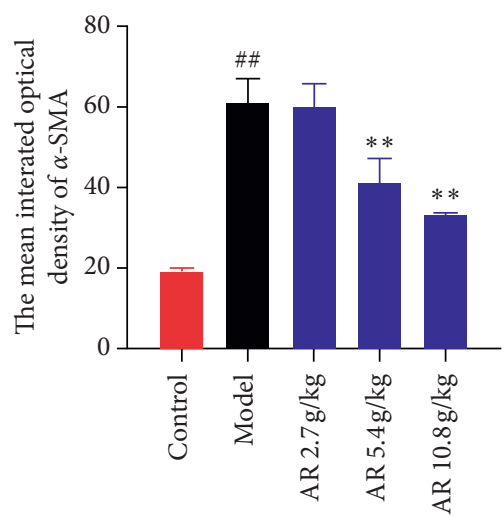

(f)

FIGURE 5: Immunohistochemistry analysis for the effect of AR on $\alpha$-SMA localization (original magnification, $\times 200$; $n=3$; (a) control group; (b) model group; (c) $2.7 \mathrm{~g} / \mathrm{kg}$ AR group; (d) $5.4 \mathrm{~g} / \mathrm{kg}$ AR group; (e) $10.8 \mathrm{~g} / \mathrm{kg}$ AR group). (f) Quantitative analysis of $\alpha$-SMA localization by immunohistochemistry.

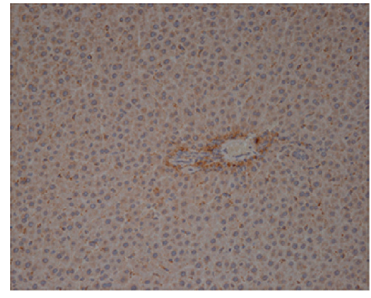

(a)

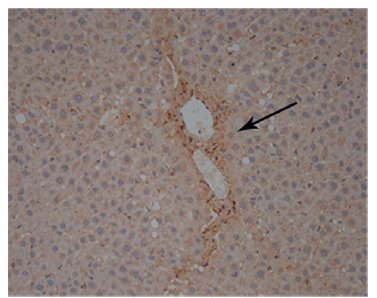

(e)

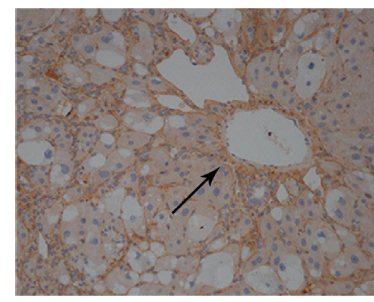

(b)

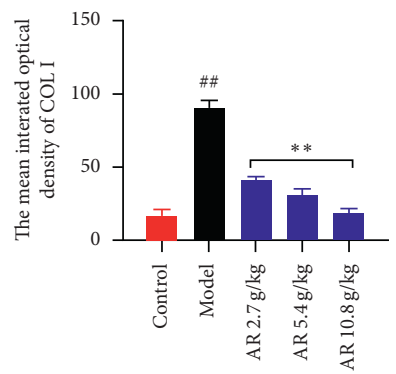

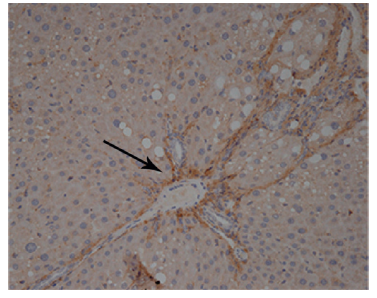

(c)

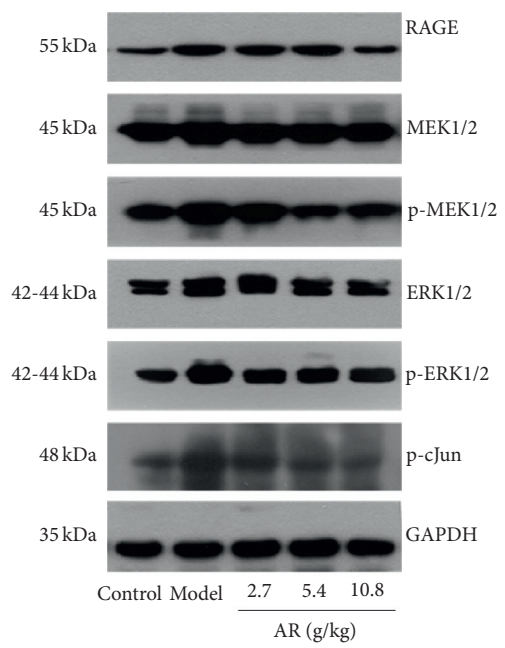

(g)

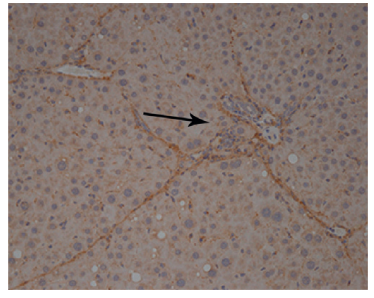

(d)

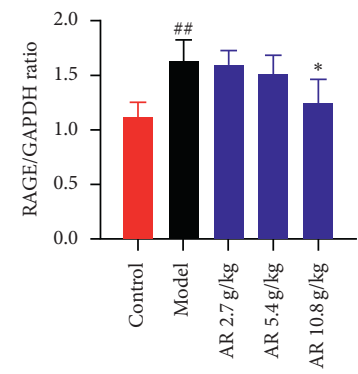

(h)

Figure 6: Continued. 


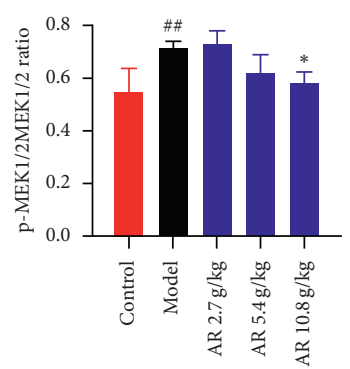

(i)

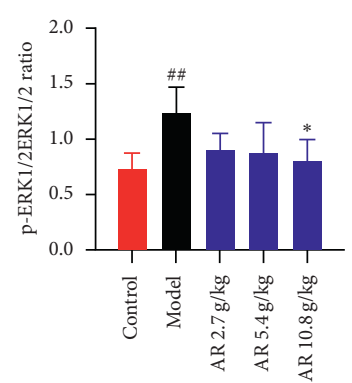

(j)

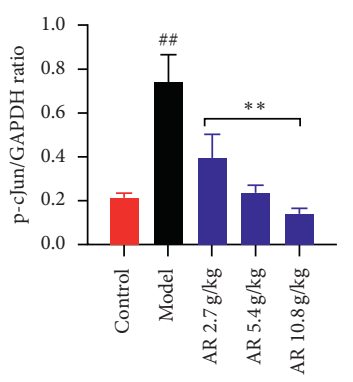

(k)

Figure 6: Effect of RA on type 1 collagen level and HMGB1/RAGE/c-Jun pathway in liver fibrosis induced by $\mathrm{CCl}_{4}$. (a-e) Immunohistochemistry analysis for the effect of AR on type 1 collagen expression (original magnification, $\times 200 ; n=3$; (a): control group; (b): model group; (c): $2.7 \mathrm{~g} / \mathrm{kg}$ AR group; (d) $5.4 \mathrm{~g} / \mathrm{kg}$ AR group; (e) $10.8 \mathrm{~g} / \mathrm{kg}$ AR group.) (f) The mean integrated optical density of COL-I. (g) Western blot images of RAGE, MEK1/2, p-MEK1/2, ERK1/2, p-ERK1/2, and p-cJun. (h) RAGE protein level. (i) p-MEK1/2 protein level. (j) p-ERK1/ 2 protein level. (k) p-cJun protein level.

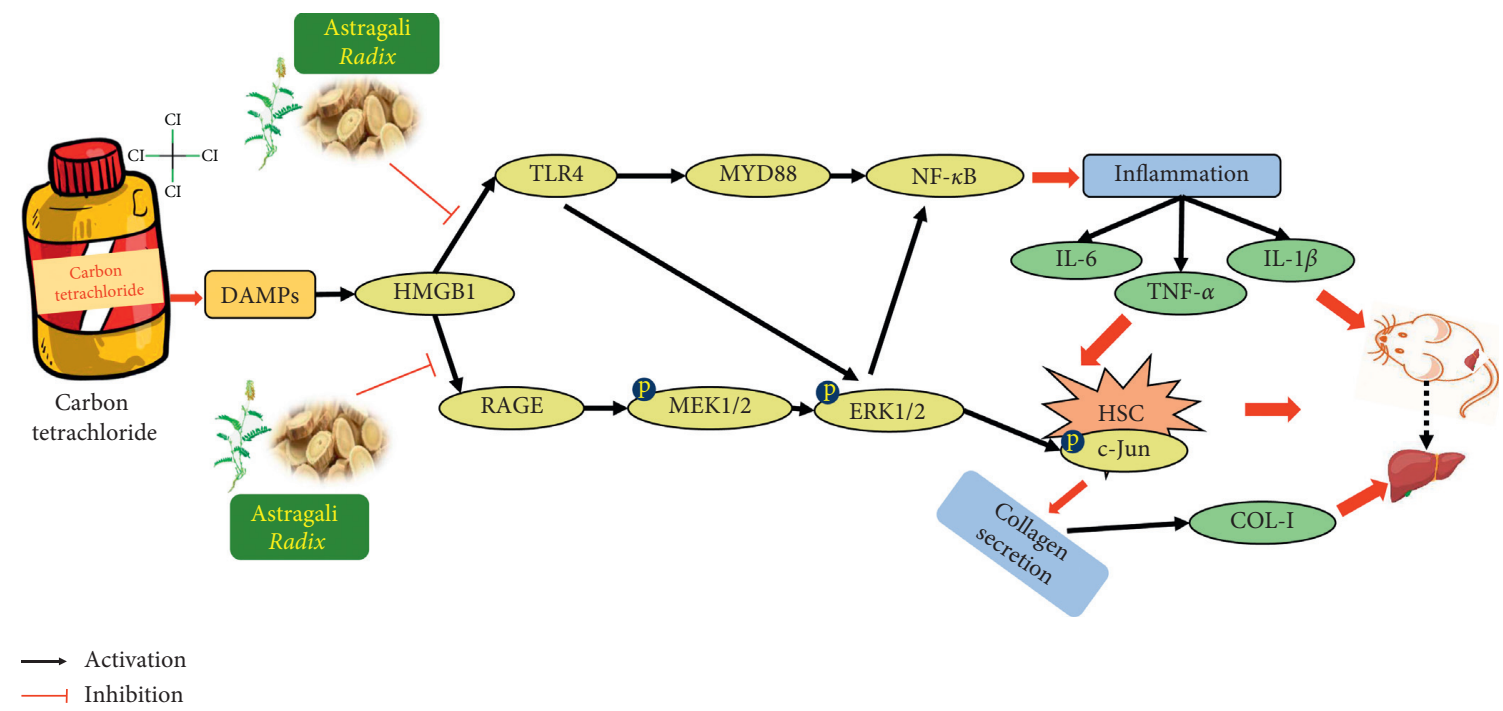

FIGURE 7: Schematic diagram of signal pathway which mediates $\mathrm{CCl}_{4}$-induced liver fibrosis and the ameliorative effects of AR on rat.

downregulate the expression of HMGB1, but also reduce the transcription of inflammatory cytokine IL- $1 \beta$, TNF- $\alpha$, and IL- 6 by regulating HMGB1/TLR4/NF- $x$ B signaling pathway. Therefore, we speculate that AR can inhibit the activation of rat HSCs. The detection of $\alpha$-SMA, a marker protein of HSCs activation, further confirmed our hypothesis: AR can significantly inhibit the activation of rat HSCs induced by $\mathrm{CCl}_{4}$ (Figure 7).

Liver fibrosis or scar deposition caused by chronic injury is similar in all forms of liver disease [41]. In the subendothelial space between hepatocytes and endothelial cells, fibrillar or type I collagen aggregates to replace the lowdensity basement membrane-like matrix containing type IV collagen. The conversion of the subendothelial matrix to fibril-rich collagen is a key event, which mediates the loss of differentiation function characteristic of progressive liver disease [42]. Myofibroblasts are the main source of type I collagen accumulated during tissue fibrosis [43]. It was found that the activation of HSCs was decreased in ARtreated group, and we speculated that the expression of type I collagen in the liver of rats would also be reduced accordingly. In addition, the latest research reports that HMGB1 activates the paracrine system and upregulates the secretion of type 1 collagen by binding to the receptor RAGE on the surface of fibroblasts, thereby triggering and/or maintaining scar formation [17]. Therefore, we detected the expression of type I collagen in the liver of rats, as well as the expression of RAGE, p-MEK1/2, p-ERK1/2, and p-c-Jun in liver tissues. The results showed that $A R$ can significantly reduce the expression of type 1 collagen in rats, which may be related to the decrease of HSCs activation and the downregulation of collagen secretion mediated by HMGB1-RAGE-c-Jun.

\section{Conclusion}

In conclusion, biochemical tests and histological assessments were used to reveal the function of AR. The study also demonstrated that $\mathrm{AR}$ was potent for downregulating the expression of HMGB1/RAGE/cJun pathway and HMGB1/ TLR4/NF- $\kappa \mathrm{B}$ pathway, thus protecting rats against $\mathrm{CCl}_{4^{-}}$ 
induced liver fibrosis. Therefore, this study will provide scientific evidence and theoretical basis for the development of $\mathrm{AR}$ as a potential drug candidate for liver fibrosis.

\section{Abbreviations}

$\begin{array}{ll}\text { AR: } & \text { Astragali Radix } \\ \text { ALT: } & \text { Alanine aminotransferase } \\ \text { AST: } & \text { Aspartate aminotransferase } \\ \mathrm{CCl}_{4}: & \text { Carbon tetrachloride } \\ \text { COL-IV: } & \text { Collagen IV } \\ \text { DAMPs: } & \text { Damage-associated molecular patterns } \\ \text { ECM: } & \text { Extracellular matrix } \\ \text { HA: } & \text { Hyaluronic acid } \\ \text { HMGB1: } & \text { High-mobility group box } 1 \\ \text { HSCs: } & \text { Hepatic stellate cells } \\ \text { H\&E: } & \text { Hematoxylin-eosin } \\ \text { IHC: } & \text { Immunohistochemistry } \\ \text { IOD: } & \text { Integrated optical density } \\ \text { LN: } & \text { Laminin } \\ \text { PCR: } & \text { Polymerase chain reaction } \\ \text { PC III: } & \text { Precollagen type III } \\ \text { RAGE: } & \text { The receptor of advanced glycation end products } \\ \text { SD: } & \text { Standard deviation } \\ \alpha \text {-SMA: } & \alpha \text {-smooth muscle actin. }\end{array}$

\section{Data Availability}

The data used to support the findings of this study are available from the corresponding author upon reasonable request.

\section{Conflicts of Interest}

The authors declare that there are no conflicts of interest.

\section{Authors' Contributions}

Jianxia Wen and Dan Wang contributed equally to this study. Jianxia Wen and Dan Wang conducted the experiments and wrote the manuscript. Ruilin Wang and Shizhang Wei analyzed the data. Yanling Zhao and Jian Wang designed the study and guided the experiment.

\section{Acknowledgments}

This work was supported by the project of Capital's Funds for Health Improvement and Research (no. 2018-2-5032).

\section{References}

[1] G. Zhang, Y. Liu, and P. Liu, "Active components from sea buckthorn (Hippophae rhamnoides L.) regulate hepatic stellate cell activation and liver fibrogenesis," Journal of Agricultural and Food Chemistry, vol. 66, no. 46, pp. 12257-12264, 2018.

[2] Y. Liu, Y. Bi, C. Mo et al., "Betulinic acid attenuates liver fibrosis by inducing autophagy via the mitogen-activated protein kinase/extracellular signal-regulated kinase pathway," Journal of Natural Medicines, vol. 73, no. 1, pp. 179-189, 2019.
[3] F. Alegre, P. Pelegrin, and A. Feldstein, "Inflammasomes in liver fibrosis," Seminars in Liver Disease, vol. 37, no. 2, pp. 119-127, 2017.

[4] D. Schuppan, M. Ashfaq-Khan, A. T. Yang, and Y. O. Kim, "Liver fibrosis: direct antifibrotic agents and targeted therapies," Matrix Biology, vol. 68-69, pp. 435-451, 2018.

[5] Y. Yoon, Y. Lee, and S. Friedman, "Antifibrotic therapies: Where are we now?" Seminars in Liver Disease, vol. 36, no. 1, pp. 087-098, 2016.

[6] F. Tacke and C. Trautwein, "Mechanisms of liver fibrosis resolution," Journal of Hepatology, vol. 63, no. 4, pp. 1038-1039, 2015.

[7] L. Campana and J. Iredale, "Regression of liver fibrosis," Seminars in Liver Disease, vol. 37, no. 1, pp. 1-10, 2017.

[8] A. Gong, R. Duan, H. Wang et al., "Evaluation of the pharmaceutical properties and value of Astragali radix," Medicines, vol. 5, no. 2, p. 46, 2018.

[9] J. Fu, Z. Wang, L. Huang et al., "Review of the botanical characteristics, phytochemistry, and pharmacology of astragalus membranaceus (huangqi)," Phytotherapy Research, vol. 28, no. 9, pp. 1275-1283, 2014.

[10] S. He, Y. Yang, X. Liu et al., "Compound astragalus and salvia miltiorrhiza extract inhibits cell proliferation, invasion and collagen synthesis in keloid fibroblasts by mediating transforming growth factor- $\beta /$ smad pathway," British Journal of Dermatology, vol. 166, no. 3, pp. 564-574, 2012.

[11] L. Li, W. Huang, S. Wang et al., "Astragaloside IV attenuates acetaminophen-induced liver injuries in mice by activating the Nrf2 signaling pathway," Molecules, vol. 23, no. 8, p. 2032, 2018.

[12] S.-Y. Yu, H.-T. Ouyang, J.-Y. Yang et al., "Subchronic toxicity studies of Radix Astragali extract in rats and dogs," Journal of Ethnopharmacology, vol. 110, no. 2, pp. 352-355, 2007.

[13] J. Li, F.-P. Wang, W.-M. She et al., "Enhanced high-mobility group box 1 (HMGB1) modulates regulatory T cells (Treg)/T helper 17 (Th17) balance via toll-like receptor (TLR)-4-interleukin (IL)-6 pathway in patients with chronic hepatitis B," Journal of Viral Hepatitis, vol. 21, no. 2, pp. 129-140, 2014.

[14] J. Li, C. Zeng, B. Zheng et al., "HMGB1-induced autophagy facilitates hepatic stellate cells activation: a new pathway in liver fibrosis," Clinical Science, vol. 132, no. 15, pp. 1645-1667, 2018.

[15] P. Qiu, L. Wang, J. Ni, and Y. Zhang, “Associations between HMGB1 gene polymorphisms and susceptibility and clinical outcomes in Chinese Han sepsis patients," Gene, vol. 687, pp. 23-29, 2019.

[16] H. Gaskell, X. Ge, and N. Nieto, "High-mobility group box-1 and liver disease," Hepatology Communications, vol. 2, no. 9, pp. 1005-1020, 2018.

[17] X. Ge, E. Arriazu, F. Magdaleno et al., "High mobility group box-1 drives fibrosis progression signaling via the receptor for advanced glycation end products in mice," Hepatology, vol. 68 , no. 6, pp. 2380-2404, 2018.

[18] S. Leppa, R. Saffrich, and W. Ansorge, "Differential regulation of c-Jun by ERK and JNK during PC12 cell differentiation," The EMBO Journal, vol. 17, no. 15, pp. 4404-4413, 1998.

[19] D. Wang, R. Li, and S. Wei, "Metabolomics combined with network pharmacology exploration reveals the modulatory properties of Astragali Radix extract in the treatment of liver fibrosis," Chinese Medicine, vol. 14, p. 30, 2019.

[20] Q. Yao, Y. Lin, X. Li, X. Shen, J. Wang, and C. Tu, "Curcumin ameliorates intrahepatic angiogenesis and capillarization of 
the sinusoids in carbon tetrachloride-induced rat liver fibrosis," Toxicology Letters, vol. 222, no. 1, pp. 72-82, 2013.

[21] Y. Yang, P. Zhang, and Y. Wang, "Hepatoprotective effect of san-cao granule on con a-induced liver injury in mice and mechanisms of action exploration," Frontiers in Pharmacology, vol. 9, p. 624, 2018.

[22] X. Feng, G. J. Zhong, D. J. Yang, L. Chen, and S. Du, "Hepatoprotective effect of Herpetospermum caudigerum wall. On carbon tetrachloride-induced hepatic fibrosis in rats," Journal of Cellular and Molecular Medicine, vol. 22, no. 7, pp. 3691-3697, 2018.

[23] E. Ramos-Tovar, E. Hernandez-Aquino, S. Casas-Grajales et al., "Stevia prevents acute and chronic liver injury induced by carbon tetrachloride by blocking oxidative stress through Nrf2 upregulation," Oxidative Medicine and Cellular Longevity, vol. 2018, Article ID 3823426, 12 pages, 2018.

[24] H.-J. Zhangdi, S.-B. Su, F. Wang et al., "Crosstalk network among multiple inflammatory mediators in liver fibrosis," World Journal of Gastroenterology, vol. 25, no. 33, pp. 4835-4849, 2019.

[25] M. Yakut, H. Özkan, M. F. Karakaya, and H. Erdal, "Diagnostic and prognostic role of serum interleukin-6 in malignant transformation of liver cirrhosis," Euroasian Journal of Hepato-Gastroenterology, vol. 8, no. 1, pp. 23-30, 2018.

[26] J.-P. Pradere, J. Kluwe, S. De Minicis et al., "Hepatic macrophages but not dendritic cells contribute to liver fibrosis by promoting the survival of activated hepatic stellate cells in mice," Hepatology, vol. 58, no. 4, pp. 1461-1473, 2013.

[27] J. Sun, Y. Wu, C. Long et al., "Anthocyanins isolated from blueberry ameliorates $\mathrm{CCl} 4$ induced liver fibrosis by modulation of oxidative stress, inflammation and stellate cell activation in mice," Food and Chemical Toxicology, vol. 120, pp. 491-499, 2018.

[28] R. Simeonova, V. M. Bratkov, M. Kondeva-Burdina, V. Vitcheva, V. Manov, and I. Krasteva, "Experimental liver protection ofn-butanolic extract ofAstragalus monspessulanusL. on carbon tetrachloride model of toxicity in rat," Redox Report, vol. 20, no. 4, pp. 145-153, 2015.

[29] Y. Zhou, X. Tong, S. Ren et al., "Synergistic anti-liver fibrosis actions of total astragalus saponins and glycyrrhizic acid via TGF- $\beta 1 /$ smads signaling pathway modulation," Journal of Ethnopharmacology, vol. 190, pp. 83-90, 2016.

[30] Y. Li, C. Wang, Y. Jin et al., "Huang-Qi San improves glucose and lipid metabolism and exerts protective effects against hepatic steatosis in high fat diet-fed rats," Biomedicine \& Pharmacotherapy, vol. 126, Article ID 109734, 2020.

[31] M. Hao, Z. Guan, Y. Gao et al., "Huang-Qi San ameliorates hyperlipidemia with obesity rats via activating brown adipocytes and converting white adipocytes into brown-like adipocytes," Phytomedicine, vol. 78, Article ID 153292, 2020.

[32] M. Ryu, E. H. Kim, M. Chun et al., "Astragali Radix elicits anti-inflammation via activation of MKP-1, concomitant with attenuation of p38 and Erk," Journal of Ethnopharmacology, vol. 115, no. 2, pp. 184-193, 2008.

[33] Y. S. Lee, O. K. Han, C. W. Park et al., "Pro-inflammatory cytokine gene expression and nitric oxide regulation of aqueous extracted Astragali radix in RAW 264.7 macrophage cells," Journal of Ethnopharmacology, vol. 100, no. 3, pp. 289-294, 2005.

[34] Y. Koyama and D. A. Brenner, "Liver inflammation and fibrosis," Journal of Clinical Investigation, vol. 127, no. 1, pp. 55-64, 2017.

[35] S. Mihm, "Danger-associated molecular patterns (DAMPs): molecular triggers for sterile inflammation in the liver,"
International Journal of Molecular Sciences, vol. 19, no. 10, p. 3104, 2018.

[36] P. Huebener, J.-P. Pradere, C. Hernandez et al., "The HMGB1/ RAGE axis triggers neutrophil-mediated injury amplification following necrosis," Journal of Clinical Investigation, vol. 125, no. 2, pp. 539-550, 2015.

[37] A. Vicentino, V. Carneiro, and D. Allonso, "Emerging role of HMGB1 in the pathogenesis of schistosomiasis liver fibrosis," Frontiers in Immunology, vol. 9, no. 1979, 2018.

[38] T. Trotta, C. Porro, and R. Calvello, "Biological role of toll-like receptor-4 in the brain," Journal of Neuroimmunology, vol. 268, no. 1-2, pp. 1-12, 2014.

[39] Z. Zhang, Q. Liu, M. Liu et al., "Upregulation of HMGB1TLR4 inflammatory pathway in focal cortical dysplasia type II," Journal of Neuroinflammation, vol. 15, no. 1, p. 27, 2018.

[40] T. Tsuchida and S. L. Friedman, "Mechanisms of hepatic stellate cell activation," Nature Reviews Gastroenterology \& Hepatology, vol. 14, no. 7, pp. 397-411, 2017.

[41] S. L. Friedman, "Molecular regulation of hepatic fibrosis, an integrated cellular response to tissue injury," Journal of Biological Chemistry, vol. 275, no. 4, pp. 2247-2250, 2000.

[42] E. Olaso, K. Ikeda, F. J. Eng et al., "DDR2 receptor promotes MMP-2-mediated proliferation and invasion by hepatic stellate cells," Journal of Clinical Investigation, vol. 108, no. 9, pp. 1369-1378, 2001.

[43] N. C. Henderson, T. D. Arnold, Y. Katamura et al., "Targeting of $\alpha \mathrm{v}$ integrin identifies a core molecular pathway that regulates fibrosis in several organs," Nature Medicine, vol. 19, no. 12 , pp. $1617-1624,2013$. 